\title{
ARTICLE OPEN \\ Real-world heart rate norms in the Health eHeart study
}

Robert Avram (D) ${ }^{1}$, Geoffrey H. Tison (D) ${ }^{1}$, Kirstin Aschbacher ${ }^{1}$, Peter Kuhar (D) $^{2}$, Eric Vittinghoff ${ }^{3}$, Michael Butzner ${ }^{1}$, Ryan Runge ${ }^{1}$, Nancy Wu ${ }^{1}$, Mark J. Pletcher ${ }^{3}$, Gregory M. Marcus ${ }^{1}$ and Jeffrey Olgin ${ }^{1}$

Emerging technology allows patients to measure and record their heart rate (HR) remotely by photoplethysmography (PPG) using smart devices like smartphones. However, the validity and expected distribution of such measurements are unclear, making it difficult for physicians to help patients interpret real-world, remote and on-demand HR measurements. Our goal was to validate HRPPG, measured using a smartphone app, against HR-electrocardiogram (ECG) measurements and describe out-of-clinic, real-world, HR-PPG values according to age, demographics, body mass index, physical activity level, and disease. To validate the measurements, we obtained simultaneous HR-PPG and HR-ECG in 50 consecutive patients at our cardiology clinic. We then used data from participants enrolled in the Health eHeart cohort between 1 April 2014 and 30 April 2018 to derive real-world norms of HR-PPG according to demographics and medical conditions. HR-PPG and HR-ECG were highly correlated (Intraclass correlation $=$ 0.90). A total of 66,788 Health eHeart Study participants contributed 3,144,332 HR-PPG measurements. The mean real-world HR was $79.1 \mathrm{bpm} \pm 14.5$. The 95 th percentile of real-world HR was $\leq 110$ in individuals aged $18-45, \leq 100$ in those aged $45-60$ and $\leq 95 \mathrm{bpm}$ in individuals older than 60 years old. In multivariable linear regression, the number of medical conditions, female gender, increasing body mass index, and being Hispanic was associated with an increased HR, whereas increasing age was associated with a reduced HR. Our study provides the largest real-world norms for remotely obtained, real-world HR according to various strata and they may help physicians interpret and engage with patients presenting such data.

npj Digital Medicine (2019)2:58; https://doi.org/10.1038/s41746-019-0134-9

\section{INTRODUCTION}

Heart rate (HR) is a readily available vital sign that holds important prognostic information. Generally, lower HR has been associated with lower all-cause and cardiovascular mortality. ${ }^{1-5}$ Several studies, as well as expert consensus, indicate that the normal adult resting $\mathrm{HR}$ values lie between 60 and 90 beats per minute (bpm) ${ }^{1-3}$ and the American Heart Association defines the normal sinus HR as between 60 and $100 \mathrm{bpm} .{ }^{3}$ However, these commonly accepted norms are derived using in-clinic recorded HR which may not be representative of the real-world, outside of a healthcare institution, remotely obtained measurements that are commonly recorded by a growing number of consumer devices. For example, clinic measured data can be artificially increased in a similar phenomenon to "white-coat hypertension" ${ }^{4}$ or by an increased adrenergic reaction to the clinical settings. ${ }^{5}$ In addition, these measurements do not account for health status, cardiovascular fitness, gender, or racial differences. Moreover, ambulatory heart rate has been found to be a stronger predictor for all-cause mortality than in-clinic resting heart rate, yet this real-world measurement is infrequently obtained. ${ }^{6}$

Recently, photoplethysmography (PPG) technology has become nearly ubiquitous in smartphones and wearable sensors (such as activity trackers or smartwatches), providing both an opportunity to measure real-world HR while increasing the importance to understand the accuracy and the normal HR values obtained by these types of ambulatory measurement. ${ }^{7,8}$ In addition, physicians are increasingly being asked by patients to interpret HR values recorded remotely by patient devices. ${ }^{9}$ However, in this setting it is unclear whether traditional clinic-derived normal values adequately represent remotely recorded real-world data. ${ }^{9}$

The Health eHeart Study, an online Framingham-like cohort, has collected a large number of HR measurements over time from study participants using PPG-enabled smartphone technology. The goals of this study were to (i) validate HR-PPG measurements against a gold-standard electrocardiographic HRelectrocardiogram (ECG) measurement, and (ii) provide realworld HR-PPG ranges according to age, time, demographics, comorbidities and chronotropic medication usage, and (iii) identify predictors of real-world HR-PPG and heart rate variability (HRV).

\section{RESULTS}

Smartphone-based PPG validation

We validated the HR-PPG measurement in 50 consecutive participants seen at the UCSF general cardiology clinic who had a 12-lead ECG performed (10 s recording) with simultaneous PPG signals recorded (Supplementary Figs. 1 and 2). These patients were older $64.0 \pm 13.1$ (vs $43.4 \pm 14.8$ in our full HR data set; $p<$ $0.0005)$, male $(66.0 \%$ vs $52.3 \% ; p<0.00005)$ and they had a higher prevalence of diabetes, hypercholesterolemia, hypertension, and arrhythmia than that in our full HR data set. There were 21 abnormal ECGs (five atrial fibrillation, two atrial flutter, three left bundle branch block, three frequent premature ventricular

\footnotetext{
${ }^{1}$ Division of Cardiology, Department of Medicine, and the Cardiovascular Research Institute, University of California, San Francisco, Cardiology (San Francisco, CA, United States),

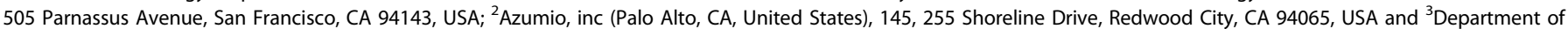
Epidemiology and Biostatistics, University of California San Francisco (San Francisco, CA, United States), 505 Parnassus Avenue, San Francisco, CA 94143, USA Correspondence: Jeffrey Olgin (Jeffrey.olgin@ucsf.edu)
}

Received: 18 February 2019 Accepted: 30 May 2019

Published online: 25 June 2019 
complex, one frequent premature atrial complex, one sinus tachycardia, six ventricular pacemaker) and 29 normal ECGs with normal sinus rhythm. The HR-PPG values had very good intraclass correlation (ICC) with HR-PPG (0.90 overall; 0.88 for irregular rhythms; and 1.00 for regular rhythms) with a median absolute HR difference between both recordings of $2.7 \mathrm{bpm}$ (6.9) (Supplementary Fig. 3A). The median difference between the two signals in successive R-R interval measurements was $12.5 \mathrm{~ms}(23.4)$ and the ICC between signals was very high ( 1.00 overall, 0.99 for irregular rhythms and 1.00 for regular rhythms) (Supplementary Fig. 3B). The Bland-Altman plots showed no evidence of trends in either the bias or the dispersion of the differences, at low, normal, or high HR values.

\section{Health eHeart study sample}

A total of 66,788 Health eHeart Study participants contributed 3,144,332 HR-PPG measurements between April 12014 and 30 April 2018, forming our "full HR data set". Of these, 33,344 (1.06\%) measurements were excluded for being outside of biological ranges (Supplementary Fig. 4). In our full HR data set, mean age was $43.3 \pm 14.8$ years and $47.0 \%$ of our participants were female (Table 1). The BMI was $27.5 \pm 5.8 \mathrm{~kg} / \mathrm{m}^{2}$ and participants walked on average $3491.1 \pm 3345.4$ steps per day, as measured by their smartphone. Slightly less than half of the participants were healthy, having reported no medical condition ( $n=25,408$, 48.3\%). The most prevalent medical conditions were hypertension, hypercholesterolemia and presence of arrhythmia (Table 1). In addition, 2412 (6.9\%) users were treated with beta blockers and $435(1.3 \%)$ were on non-dihydropyridine CCBs, amiodarone, or inhaled beta agonists (Table 1).

\section{Description of our HR data sets}

A total of 40,572 measurements from 8046 participants met our definition for inclusion in the "known resting HR data set". After obtaining real-world user-specific HR-PPG, the geometric mean HR-PPG in our "known resting HR" data set was $2.8 \mathrm{bpm}$ higher compared with our "full HR data set" and had a higher spread of values (81.8 \pm 19.6 (95\% percentile interval: $52.5-132.1)$ vs $79.0 \pm$ 14.5 (95\% percentile interval: $54.5-110.8$ ), respectively; $p<0.0005$ (Supplementary Tables 2A-C, 4 and Fig. 5).

In our "full HR data set", real-world HR varied significantly over the day with the lowest values observed between midnight and 5 $\mathrm{AM}$ (nadir at $5 \mathrm{am} ; 75.8 \pm 22.4$ ) and the highest values observed between $5 \mathrm{AM}$ and 5 PM (peak at 5 pm; $82.3 \pm 23.7 ; p<0.0005$ ) (Supplementary Fig. 6). The HR was higher during weekdays compared with weekends (79.1 \pm 17.6 vs. $78.4 \pm 17.1 ; p<0.0005)$. Similarly, the HRV was highest between 6 am and $12 \mathrm{pm}(14.9 \pm$ 10.6) and lowest between $6 \mathrm{pm}$ and $12 \mathrm{am}(12.5 \pm 9.6 ; p<0.0005$ compared with $6 \mathrm{am}-12 \mathrm{pm}$ ). It was also higher during weekdays than weekends $(13.7 \pm 10.7$ vs. $13.3 \pm 10.4 ; p<0.0005)$ (Supplemental Table 1). Finally, the highest average HR was observed during winter $(79.1 \pm 16.7)$, whereas the lowest HR was observed during fall (78.4 $\pm 16.5 ; p<0.0005$ compared with winter). HRV was highest during summer $(15.0 \pm 10.2)$ and fell to the lowest levels during winter (14.1 $\pm 10.0 ; p<0.0005$ compared with summer) and spring (14.1 $\pm 10.2 ; p<0.0005$ compared with summer).

Heart rate according to age, demographics, step count, comorbidities, and medications

We describe variations in HR-PPG according to various factors within the subgroup who reported no medical conditions $(n=$ 25,$408 ; 48.3 \%$ of users and $n=1,103,570$ measurements). These "healthy individuals" were younger ( $37.7 \pm 13.0$ vs. $43.4 \pm 14.8 ; p<$ $0.0005)$, with a higher proportion of females $(50.2 \%$ vs $47.0 \%$; $p<$ $0.0005)$, similar racial/ethnic group composition, similar step counts and a lower BMI $(26.1 \pm 5.3$ vs. $28.7 \pm 6.0)$ when compared with our "full HR data set". In healthy individuals, average resting HR-PPG decreased from $81.6 \pm 14.0$ in those aged $18-20$ to $74.2 \pm$ 12.7 in those aged $71-80(p<0.0005)$ (Table 2 and Fig. 1a). The 95th percentile of real-world HR was uniformly under $100 \mathrm{bpm}$ after 45 years of age reaching approximately $95 \mathrm{bpm}$ at 61 years of age. Females had on average a HR-PPG $4.4 \mathrm{bpm}$ higher than men (Fig. 1b). As age increased, the $95 \% \mathrm{Cl}$ of HR-PPG values narrowed in women more than men (18-20 years, women 56.8-113.1 bpm, men 54.4-106.2 bpm; vs age 60-70, women 56.3-103.7 bpm, men 52.4-101.9 bpm, Fig. 1b and Supplementary Table 2B, C). African Americans and Asians had the highest HRPPG (81.4 $\pm 14.0 \mathrm{bpm}$ and $79.2 \pm 14.3 \mathrm{bpm}$, respectively) and nonHispanic White had the lowest HR-PPG (75.9 $\pm 14.5 \mathrm{bpm})$ (Table 2). HR-PPG increased with BMI from $74.9 \pm 16.5 \mathrm{bpm}$ in individuals with a BMl of $18.5-25 \mathrm{~kg} / \mathrm{m}^{2}$ to $80.1 \pm 13.3 \mathrm{bpm}$ in those with $\mathrm{BMI} \geq 30 \mathrm{~kg} / \mathrm{m}^{2}(p<0.0005)$. A negative relationship between HRPPG and daily step counts was observed, with the HR-PPG values significantly decreasing from $80.0 \pm 13.5 \mathrm{bpm}$ in sedentary participants walking 2001-4000 steps per day to 78.0 \pm 13.9 bpm in active participants walking 8001-12,000 steps per day $(p<$ 0.0005 ) (Table 2, Fig. 1c and Supplementary Table 3). We observed no significant difference in the real-world HR-PPG between groups above an average of 8001 steps per day. Height was a predictor of reduced heart rate where for every 1 centimeter, the HR was reduced by $0.23 \mathrm{bpm}$. In univariable analysis, age, height, and number of steps were negative predictors of heart rate and female gender, BMI, Asian race and multi-ethnicity were predictors of an increased heart rate (Supplementary Table 5). Weight was not a significant predictor of heart rate. In a multivariable analysis, age was significantly associated with lowered heart rate, whereas female gender, BMI, Hispanic ethnicity and the number of medical conditions had a positive relationship with HR-PPG, however step counts were not a significant independent predictor of HR-PPG (Table 4; Model 1).

An increase in average daily steps was associated with higher $H R V$, whereas an increase in age and BMI were significant predictors of a lower HRV (Table 5; Model 1). In Model 2, looking at age, gender, disease state, and medications, we observed a lower HRV for increasing age, female gender, hypertension, and an increase in HRV for Hispanic ethnicity, sleep apnea, and users on CCB (Table 5; Model 2). No other medical conditions or medications were significant predictors of HRV in our cohort.

Participants who reported having at least one medical condition ( $n=27,958)$ contributed two thirds $(2,007,418)$ of the HR-PPG measurements. They had a higher HR-PPG compared with those who reported no medical conditions $(79.6 \pm 14.2 \mathrm{bpm}$ vs $77.6 \pm$ $14.6 \mathrm{bpm}, p<0.0005)$, even after adjusting for age. Those suffering from any medical condition, except coronary artery disease, prior myocardial infarction (MI) and hypercholesterolemia had a significantly higher HR-PPG than those without the condition (Table 3). Once adjusting for age, HR was higher in those with hypercholesterolemia and coronary artery disease. The highest difference was observed in those with diabetes $(82.6 \pm 14.1 \mathrm{bpm}$ vs $78.3 \pm 14.5 \mathrm{bpm}$ without diabetes), followed by those with COPD (82.5 \pm 13.9 bpm with vs $78.3 \pm 14.5$ bpm without COPD). In multivariable analysis (Table 4, Model 2), female gender, all races/ ethnicities other than non-Hispanic White and participants suffering from hypertension, hypercholesterolemia, diabetes, arrhythmia, sleep apnea, COPD, and asthma were independent predictors of a higher HR-PPG, whereas increasing age was a predictor of a lower HR-PPG. All medical conditions were associated with a higher HR-PPG, when adjusting for age, gender, and beta-blocker use (Supplementary Table 6). Similarly, asthma and COPD were associated with a higher HR-PPG after adjustment for beta-agonist use. 


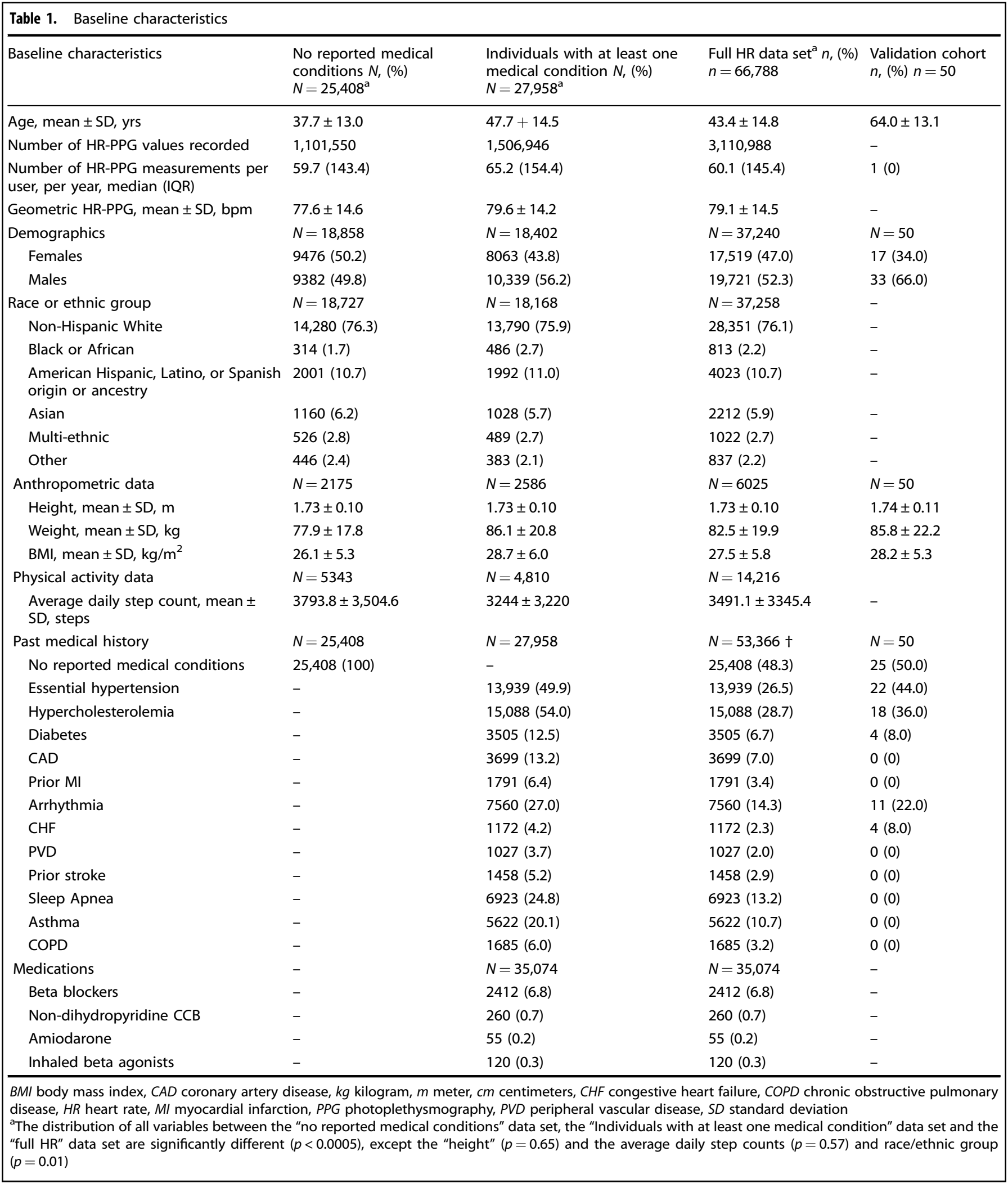

\section{DISCUSSION}

As the use of smartphone sensors and wearable devices provides data on cardiovascular parameters such as HR, physicians are increasingly expected to help patients interpret the results of these readings; however, existing norms derived from controlled, clinical settings may not reflect the range of $\mathrm{HR}$ values occurring in real-world conditions. Our validation demonstrated that smartphone-based HR-PPG strongly correlates with HR from the gold-standard ECG. This study provides the first and largest-scale description of real-world HR values derived from smartphone 
Table 2. Heart rate according to age, gender, race, and step count in healthy participants

\begin{tabular}{|c|c|c|c|}
\hline $\begin{array}{l}\text { Baseline } \\
\text { characteristics }\end{array}$ & $\begin{array}{l}\text { Number of } \\
\text { participants } \\
\text { contributing at least } \\
\text { one HR-PPG } \\
\text { measurement } N(\%)\end{array}$ & $\begin{array}{l}\text { Number of HR-PPG } \\
\text { measurements } \\
\text { Median (IQR) }\end{array}$ & $\begin{array}{l}\text { Geometric } \\
\text { mean } \mathrm{HR} \pm \mathrm{SD}^{\mathrm{a}}\end{array}$ \\
\hline Age stratum & $N=25,280$ & & \\
\hline $18-20$ & $2197(8.7)$ & $11.0(20.0)$ & $81.6 \pm 14.0$ \\
\hline $21-30$ & $6558(25.9)$ & $15.0(30.0)$ & $80.2 \pm 14.8$ \\
\hline $31-40$ & 6480 (25.6) & $18.0(37.0)$ & $78.5 \pm 15.1$ \\
\hline $41-50$ & $5620(22.2)$ & $21.0(45.0)$ & $75.3 \pm 14.3$ \\
\hline $51-60$ & $3058(12.1)$ & $23.0(54.0)$ & $73.9 \pm 13.5$ \\
\hline $61-70$ & $1173(4.6)$ & $26.0(54.0)$ & $73.0 \pm 12.7$ \\
\hline $71-80$ & $194(0.8)$ & $29.5(72.3)$ & $74.2 \pm 11.1$ \\
\hline$>80$ & $13(0.1)$ & $36.0(80.0)$ & $78.1 \pm 16.5$ \\
\hline Gender & $N=18,858$ & & \\
\hline Females & $9476(50.2)$ & $18.0(38)$ & $79.3 \pm 14.0$ \\
\hline Males & $9382(49.8)$ & $21.0(45)$ & $73.8 \pm 14.5$ \\
\hline $\begin{array}{l}\text { Race or } \\
\text { ethnic group }\end{array}$ & $N=18,727$ & & \\
\hline Non-Hispanic White & $14,280(76.3)$ & $20.0(43.0)$ & $75.9 \pm 14.5$ \\
\hline $\begin{array}{l}\text { Black or African } \\
\text { American }\end{array}$ & $314(1.7)$ & $12.0(36.8)$ & $81.4 \pm 14.0$ \\
\hline $\begin{array}{l}\text { Hispanic, Latino, or } \\
\text { Spanish origin or } \\
\text { ancestry }\end{array}$ & $2001(10.7)$ & $18.0(37.0)$ & $78.6 \pm 14.3$ \\
\hline Asian & $1160(6.2)$ & $17.0(38.8)$ & $79.2 \pm 14.3$ \\
\hline Multi-ethnic & $526(2.8)$ & $20.0(34.0)$ & $78.1 \pm 14.4$ \\
\hline $\begin{array}{l}\text { Other/prefer not to } \\
\text { disclose }\end{array}$ & $440(2.4)$ & $15(34.0)$ & $78.2+13.0$ \\
\hline BMI & $N=2175$ & & \\
\hline$<18.5$ & 58 & $21.0(54.2)$ & $77.9 \pm 14.7$ \\
\hline$\geq 18.5-25$ & 1027 & $29.0(62.0)$ & $74.9 \pm 16.5$ \\
\hline $25-30$ & 686 & $29.0(69.0)$ & $73.0 \pm 15.1$ \\
\hline$\geq 30$ & 404 & $24.5(58.8)$ & $80.1 \pm 13.3$ \\
\hline $\begin{array}{l}\text { Daily step count } \\
\text { stratum }\end{array}$ & $N=5343$ & & \\
\hline $100-2000$ & $3646(68.2)$ & $36.0(66.0)$ & $78.9 \pm 14.4$ \\
\hline $2001-4000$ & $826(15.4)$ & $35.0(72.0)$ & $80.0 \pm 13.5$ \\
\hline $4001-6000$ & $422(7.9)$ & $39.5(82.0)$ & $79.0 \pm 14.8$ \\
\hline $6001-8000$ & $241(4.5)$ & $31.0(77.0)$ & $77.6 \pm 16.4$ \\
\hline $8001-10,000$ & $113(2.1)$ & $45.0(80.0)$ & $77.9 \pm 17.3$ \\
\hline $10,001-12,000$ & $64(1.2)$ & $23.0(69.3)$ & $78.0 \pm 13.9$ \\
\hline $12,001-14,000$ & $31(0.6)$ & $31.0(37.0)$ & $81.9 \pm 15.0$ \\
\hline
\end{tabular}

$H R-P P G$ heart rate as measured using photoplethysmography, $B M I$ body mass index, $H R$ heart rate, $S D$ standard deviation

${ }^{a}$ All intergroup comparisons were significant $(p<0.0005)$

HR-PPG measurements from 66,788 individuals who provided over three million data points over a 3-year period. Furthermore, we describe how demographic and medical factors affect these norms, including age, gender, race/ethnicity, anthropometric characteristics, physical activity, and disease state. These data provide reference ranges of real-world HR for patients and physicians and establish the foundation for future research, in which real-world HR might become an outcome for large-scale studies to understand the evolution of disease at an international scale.

Higher baseline HR has been shown to be an important prognostic factor, with higher HR associated with increased allcause and cardiovascular mortality. ${ }^{1-5,10}$ However, previous studies have shown that HRs measured in clinical settings may not be representative of real-world HR and may be biased by the particular clinical conditions for which the ECGs were ordered., ${ }^{1,3}$ For example, a "white-coat" effect can increase $\mathrm{HR}^{1}{ }^{11}$ leading to false elevation. Furthermore, ambulatory, real-world HR, has been found to correlate significantly more with mortality than resting

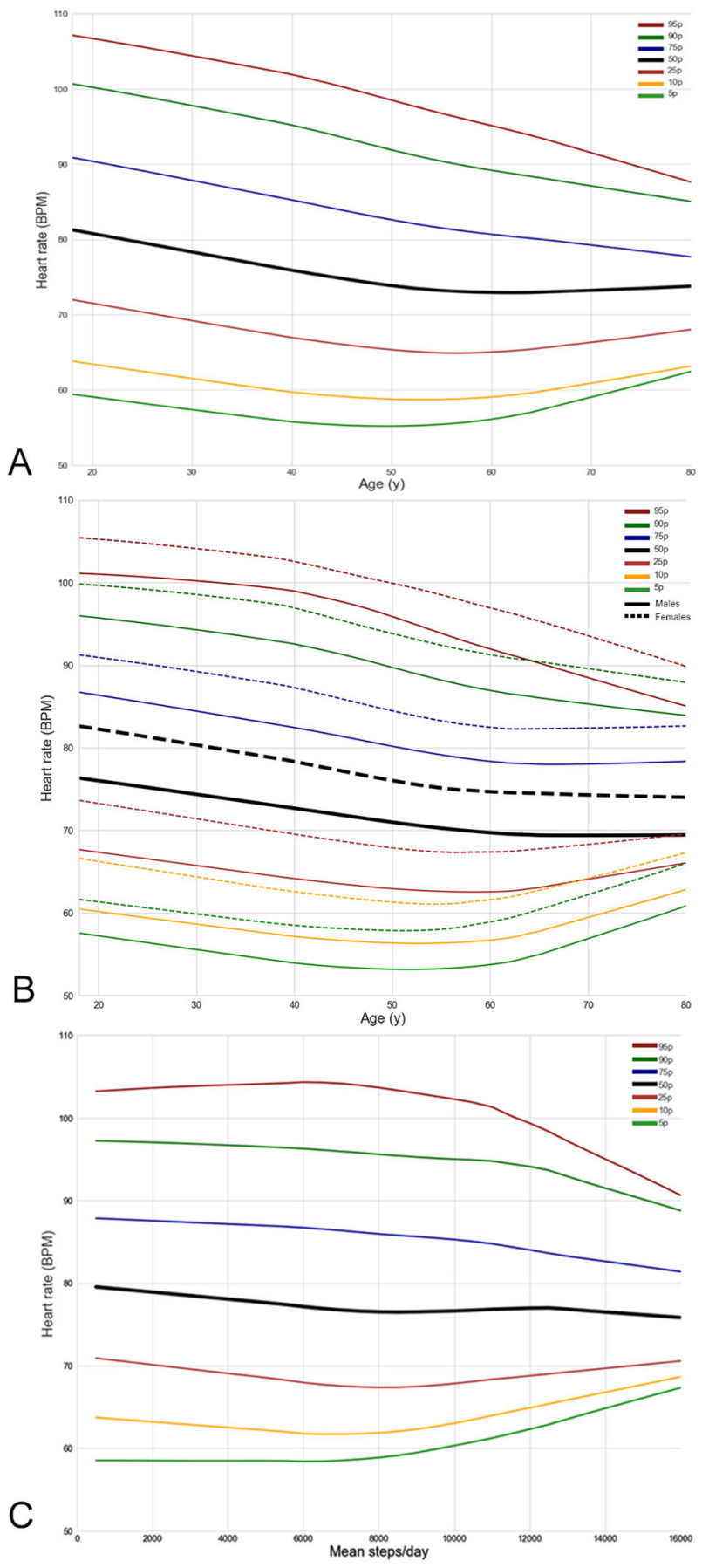

Fig. 1 Percentile graph of average real-world HR-PPG. a Percentile graph of average real-world HR-PPG according to the age. b Percentile graph of average real-world HR-PPG according to the gender c Percentile graph of average real-world HR-PPG according to the step counts

heart rate obtained in the clinical setting ${ }^{12}$ suggesting the need to update HR norms to reflect real-world, remotely obtained values. ${ }^{6}$ The median HR-PPG of 77.6 in healthy individuals of our cohort was higher than the median HR of $68.0 \mathrm{bpm}$ described by Mason et al., ${ }^{3}$ who studied 79,743 ambulatory subjects that had a single ECG done in a clinical setting. As we averaged across multiple measurements per user (median of 60.0 measurements per user per year), our data may provide a better approximation of the average real-world HR-PPG compared with a single measurement. The NHANES study followed 20,749 Adults living in the 


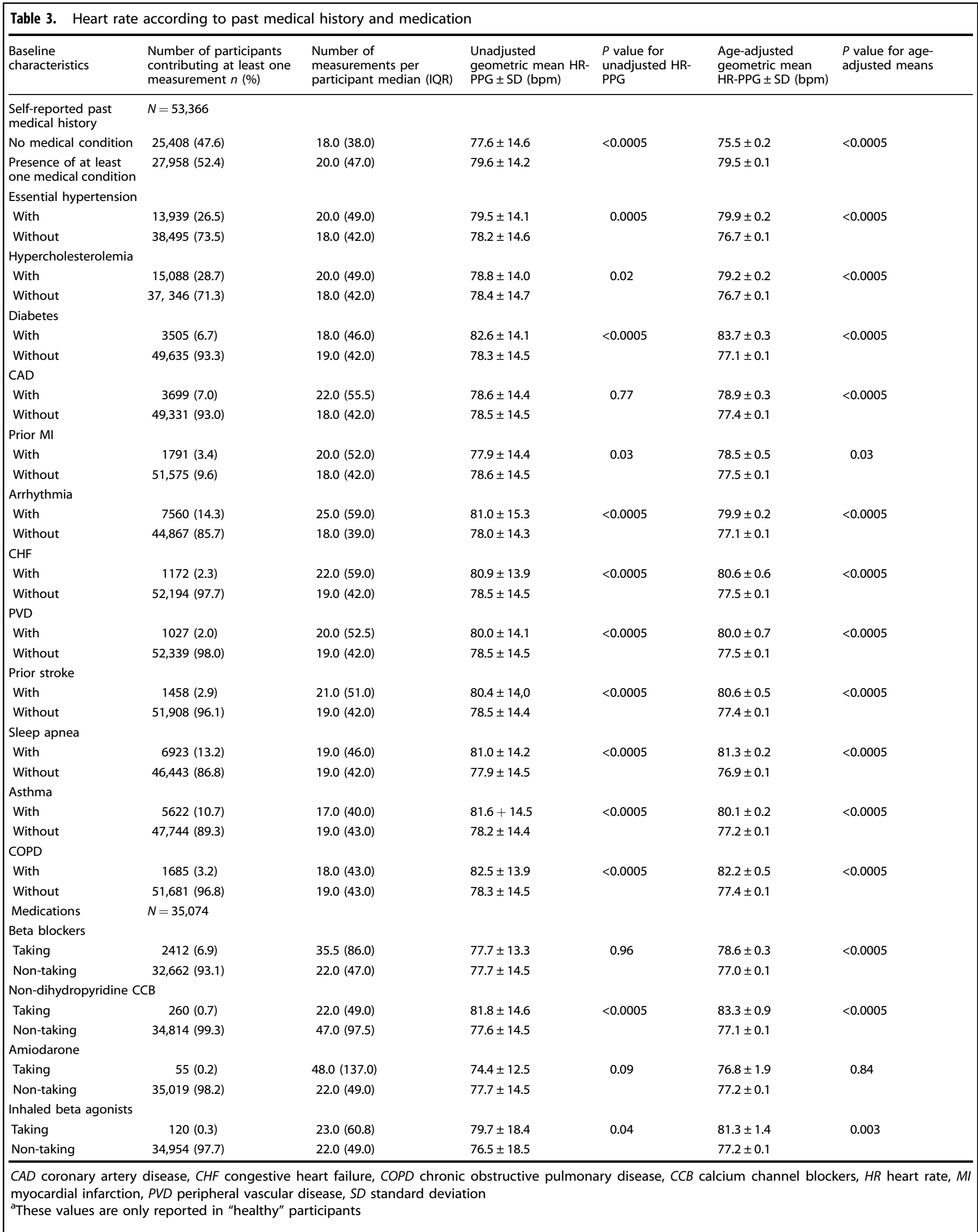


Table 4. Multivariable regression models for mean heart rate

Model 1. Multivariable linear regression describing the relationship between age, gender, daily step count, body mass index and number of diseases with geometric mean HR-PPG, in 1400 participants $^{\mathrm{a}}$

Model 2. Multivariable linear regression describing the relationship between age, gender, disease state and medications with geometric mean HR-PPG, in 31,393 participants $^{\mathrm{b}}$

\begin{tabular}{|c|c|c|c|c|c|c|c|}
\hline Variable & Coefficient & $95 \% \mathrm{Cl}$ & $p$ value & Variable & Coefficient & $95 \% \mathrm{Cl}$ & $p$ value \\
\hline Age, per 10-year increments & -2.59 & $-3.18-2.01$ & $<0.0005$ & Age, per 10-year increments & -1.93 & $-2.05-1.81$ & $<0.0005$ \\
\hline Gender, for females & 4.00 & $2.40-5.57$ & $<0.0005$ & Gender, for females & 4.28 & $3.97-4.59$ & $<0.0005$ \\
\hline Race or ethnic group & & & $<0.0005$ & Race or ethnic group & & & $<0.0005$ \\
\hline Non-Hispanic White & Ref. & - & - & Non-Hispanic White & Ref. & - & \\
\hline Black or African American & 0.57 & $-4.21-5.55$ & 0.79 & Black or African American & 3.00 & $1.94-4.06$ & $<0.0005$ \\
\hline $\begin{array}{l}\text { Hispanic, Latino, or Spanish origin or } \\
\text { ancestry }\end{array}$ & 0.37 & $-2.42-3.17$ & 0.79 & $\begin{array}{l}\text { Hispanic, Latino, or Spanish origin } \\
\text { or ancestry }\end{array}$ & 1.87 & $1.38-2.36$ & $<0.0005$ \\
\hline Asian & 5.65 & $2.30-8.99$ & 0.001 & Asian & 3.32 & $2.67-3.96$ & $<0.0005$ \\
\hline Multi-ethnic & -0.47 & $-4.98-4.03$ & 0.84 & Multi-ethnic & 1.25 & $0.32-2.18$ & $<0.0005$ \\
\hline Other/prefer not to disclose & -0.02 & $-7.27-7.23$ & 1.00 & Other/prefer not to disclose & 1.82 & $0.81-2.83$ & $<0.0005$ \\
\hline $\begin{array}{l}\text { Number of medical conditions, per } \\
\text { medical condition }\end{array}$ & 0.83 & $0.22-1.44$ & 0.01 & Diabetes & 4.48 & $3.78-5.19$ & $<0.0005$ \\
\hline Average daily steps, per 1000 steps & 0.03 & $-0.19-0.25$ & 0.79 & Arrhythmia & 1.65 & $1.19-2.11$ & $<0.0005$ \\
\hline \multirow[t]{14}{*}{ BMI } & 0.21 & $0.08-0.34$ & 0.001 & Sleep apnea & 3.67 & $3.19-4.16$ & $<0.0005$ \\
\hline & & & & $C C B$, non-dihydropyridine & 4.11 & $2.33-5.89$ & $<0.0005$ \\
\hline & & & & COPD & 2.49 & $1.50-3.48$ & $<0.0005$ \\
\hline & & & & Asthma & 1.51 & $0.99-2.03$ & $<0.0005$ \\
\hline & & & & Hypertension & 1.83 & $1.43-2.23$ & $<0.0005$ \\
\hline & & & & Hypercholesterolemia & 1.35 & $0.98-1.72$ & $<0.0005$ \\
\hline & & & & Beta agonists & 2.58 & $-0.10-5.26$ & 0.06 \\
\hline & & & & Beta blockers & -0.48 & $-1.12-0.16$ & 0.14 \\
\hline & & & & Amiodarone & -2.07 & $-5.95-1.81$ & 0.30 \\
\hline & & & & CAD & -0.28 & $-1.12-0.56$ & 0.51 \\
\hline & & & & Prior MI & -0.25 & $-1.43-0.94$ & 0.68 \\
\hline & & & & PVD & 0.28 & $-1.20-1.75$ & 0.72 \\
\hline & & & & Prior stroke & 0.64 & $-0.43-1.71$ & 0.24 \\
\hline & & & & $\mathrm{CHF}$ & 0.36 & $-1.05-1.77$ & 0.62 \\
\hline
\end{tabular}

$B M I$ body mass Index, $C A D$ coronary artery disease, $C C B$ calcium channel blockers, non-dihydropyridine, $C H F$ congestive heart failure, $C l$ confidence interval, $C O P D$ chronic obstructive pulmonary disease, $H R$ heart rate, $P P G$ photoplethysmography, $P V D$ peripheral vascular disease, $M I$ myocardial Infarction, $y$ years a'We had 1400 observations for model 1, owing to the inclusion of step counts and $\mathrm{BMI}$ as a predictor. The adjusted $R^{2}$ was $0.01 ; P<0.0005$.

${ }^{b}$ We had 31,393 observations for model 2 . The adjusted $R^{2}$ was $0.09 ; P<0.0005$

United States and described their in-clinic resting HR over 3 years. ${ }^{2}$ Compared with this study, the corresponding levels of the 5th percentile were lower in our cohort (50-55 bpm vs $60 \mathrm{bpm})$, which demonstrates a discrepancy between HR obtained in-clinic versus in a real-world setting, whereas in clinic HR tend to be trending higher than real-world $\mathrm{HR}^{2}$. Whereas our 95th percentile was similar among those $<40$ years old (104 bpm in both cohorts), among those $>40$ years old, we found a lower 95th percentile (100 bpm vs $104 \mathrm{bpm})^{2}$, which may be due to our repeat measurements taken outside of the clinic setting, decreasing variability, and minimizing any "white-coat" heart rate effect. ${ }^{11}$ Furthermore, we described the circadian evolution of real-world $\mathrm{HR}$, which allows us to interpret these values according to the time of day..$^{13}$ We observed that HR-PPG and HRV levels decline with age. Our observations suggest that the 95th percentile of realworld HR-PPG is $\leq 110$ in individuals aged $18-45$ years old, $\leq 100$ in those aged $45-60$ and $\leq 95 \mathrm{bpm}$ in individuals $>60$ years old. This decrease in maximum HR-PPG and HRV as people get older is mainly owing to a sympathetic modulation decline with aging. $^{10,14,15}$

A considerable number of epidemiologic studies have demonstrated a link between a higher HR and increasing burden of atherosclerosis ${ }^{16}$ and cardiovascular outcomes ${ }^{1,3-5,17,18}$ as well as the existence of a biological gradient between the severity of atherosclerosis and resting HR. ${ }^{19}$ Increased HR has been linked to atherosclerosis risk factors and endothelial dysfunction, plaque erosion and plaque rupture. ${ }^{20}$ Furthermore, it is acknowledged that the stress on the cardiovascular system is better investigated by real-world measurements rather than measurements obtained at rest, in a stressful clinic environment. ${ }^{6,12}$ Real-world HR is more reproducible than resting $\mathrm{HR}$ obtained in clinical setting. ${ }^{21}$ Therefore, it is possible that real-world measurements would better correlate than resting HR measurements with cardiovascular outcomes. ${ }^{12}$ Our study observed a higher HR-PPG for participants with hypercholesterolemia, hypertension, diabetes, $\mathrm{Ml}$, a prior stroke and peripheral vascular disease, all risk factors or manifestations of systemic atherosclerosis. ${ }^{1}$ Similarly, a chronic increase in sympathetic tone leading to higher $\mathrm{HR}$, as was observed in our cohort, has been described in patients with COPD, sleep apnea and asthmatic patients. ${ }^{22-25}$

In our cohort, women had a higher HR than those of men by 6 bpm, which extends prior observations to the real-world setting. ${ }^{2,14,26-29}$ It has been speculated to be owing to women having, on average, smaller stroke volumes. ${ }^{1,26,28,30}$ Differences in resting HR-PPG by race has also been previously reported in smaller studies of fewer than 170 participants. ${ }^{22,23}$ Bathula et al. $^{31}$ 
Table 5. Multivariable regression models for intra-user standard deviation of heart rate measurements

Model 1. Multivariable linear regression describing the relationship between age, gender, daily step count, body mass index and number of diseases with geometric mean HR-PPG in 1652 participants $^{a}$

\begin{tabular}{lccc}
\hline Variable & Coefficient & $95 \% \mathrm{Cl}$ & $p$-val \\
\hline Age, per 10-year increments & -0.47 & $-0.79-0.14$ & 0.01 \\
Gender, for females & 0.34 & $-0.54-1.28$ & 0.45 \\
Race or ethnic group & & & \\
Non-Hispanic White & Ref. & - & \\
Black or African American & -0.06 & $-2.77-2.64$ & 0.96 \\
Hispanic, Latino, or Spanish origin & 0.28 & $-1.27-1.83$ & 0.72 \\
or ancestry & & & \\
Asian & 0.17 & $-1.69-2.03$ & 0.86 \\
Multi-ethnic & -0.85 & $-3.35-1.64$ & 0.50 \\
Other/prefer not to disclose & -0.60 & $-4.62-3.42$ & 0.77
\end{tabular}

Number of medical conditions, per medical condition

$\begin{array}{lrrr} & 0.07 & -0.28-0.41 & 0.70 \\ \text { Average daily steps, per } 1000 \text { steps } & 0.15 & 0.03-0.28 & 0.02 \\ \text { BMI } & -0.10 & -0.18-0.03 & 0.01\end{array}$

Model 2. Multivariable linear regression describing the relationship between age, gender, disease state, and medications with intra-user standard deviation of HR-PPG, in 30,700 participants ${ }^{\mathrm{b}}$

\begin{tabular}{lccc}
\hline Variable & Coefficient & $95 \% \mathrm{Cl}$ & $p$-value \\
Age, per 10-year increments & -0.45 & $-0.53-0.37$ & $<0.0005$ \\
Gender, for females & -0.48 & $-0.69-0.27$ & $<0.0005$ \\
Race or ethnic group & & & $<0.0005$ \\
$\quad$ Non-Hispanic White & Ref. & - & \\
$\quad$ Black or African American & 0.61 & $-0.09-1.31$ & 0.09 \\
$\quad$ Hispanic, Latino, or Spanish origin or & 0.65 & $0.33-0.98$ & $<0.0005$ \\
ancestry & & & \\
$\quad$ Asian & 0.09 & $-0.34-0.52$ & 0.67 \\
Multi-ethnic & 0.19 & $-0.42-0.81$ & 0.81 \\
Other/prefer not to disclose & -0.31 & $-0.98-0.36$ & 0.36 \\
& & & \\
Diabetes & -0.28 & $-0.75-0.19$ & 0.24 \\
Arrhythmia & 0.89 & $0.59-1.19$ & $<0.0005$ \\
Sleep apnea & 0.63 & $0.30-0.95$ & $<0.0005$ \\
Non-dihydropyridine CCB & 3.92 & $2.18-5.67$ & $<0.0005$ \\
COPD & -0.22 & $-0.88-0.43$ & 0.50 \\
Asthma & -0.29 & $-0.63-0.05$ & 0.10 \\
Hypertension & -0.47 & $-0.73-0.20$ & $<0.0005$ \\
Hypercholesterolemia & -0.08 & $-0.32-0.17$ & 0.55 \\
Beta agonists & 0.10 & $-1.65-1.86$ & 0.91 \\
Beta blockers & 0.002 & $-0.419-0.423$ & 0.99 \\
Amiodarone & 1.01 & $-1.61-3.62$ & 0.45 \\
CAD & -0.13 & $0.70-0.42$ & 0.65 \\
Prior Ml & 0.26 & $-0.53-1.04$ & 0.52 \\
PVD & 0.45 & $-0.51-1.42$ & 0.36 \\
Prior stroke & 0.49 & $-0.22-1.20$ & 0.17 \\
CHF & -0.24 & $-1.17-0.69$ & 0.61 \\
\hline
\end{tabular}

$B M I$ body mass Index, $C A D$ coronary artery disease, $C C B$ calcium channel blockers, non-dihydropyridine, $C H F$ congestive heart failure, $C I$ confidence interval, COPD chronic obstructive pulmonary disease, $H R$ heart rate, $P P G$ photoplethysmography, PVD peripheral vascular disease, MI myocardial Infarction, $y$ year ${ }^{a}$ We had 142 observations for model 1, owing to the inclusion of step counts and $\mathrm{BMI}$ as a predictor. The adjusted $R^{2}$ was $0.01 ; P=0.001$

${ }^{b}$ We had 30,700 observations for model 2 . The adjusted $R^{2}$ was $0.009 ; P<0.0005$

demonstrated that on average, South Asians have $5 \mathrm{bpm}$ higher HR-PPG than Europeans, findings that seemed genetically driven and were not related to other risk factors. Our cohort extends prior literature, by demonstrating within a larger sample size that African Americans had the highest HR-PPG. These racial differences may be explained by distinct genetic phenotypes, leading to a different neural control of HR-PPG in African Americans compared with Non-Hispanic Whites. ${ }^{14}$ We also observed an increase in HR-PPG and a reduction of HRV with increasing BMI, where individuals with a BMI $\geq 30$ had a higher HR-PPG compared with their "normal weight" counterparts. These data reveal that obesity is associated with higher HRs, suggesting that weight loss may lead to lower HR and better overall health. ${ }^{18,32}$ Large-scale epidemiological studies involving 13,761 adults, demonstrated the link between an activation of the sympathetic nervous system, increased HR, and pulse pressure and BMI. ${ }^{32}$ Furthermore, we observed a "U-shaped" relationship with BMI and HR, where both underweight and overweight participants demonstrate an increase in HR compared with their 'normal weights' counterparts, complementing prior findings from the literature. ${ }^{33}$ We detected a reduction in $\mathrm{HR}$ with height, whereas the taller the person, the lower the heart rate was, extending prior findings from the literature. ${ }^{34}$ Our large sample enabled us to describe the realworld HR-PPG distribution according to daily step count strata. We observed that individuals with a higher activity level as measured by step counts had a lower HR-PPG and a higher HRV, which is consistent with prior studies. ${ }^{35-37}$ We also showed that for an increase of 5000 steps, the average resting HR-PPG decreased by $1 \mathrm{bpm}$, up to $\sim 8000$ steps/day. However, step count was not a significant predictor of reduced HR-PPG after multivariable adjustment, suggesting that the benefits of increased step counts might be difficult to disentangle from the effects of age, gender or racial differences. Our findings extend prior findings by being the largest cohort of real-world HR measures to date, reinforcing the notion that individual characteristics such as age, gender, ethnicity, step counts, and BMI should be taken into account when interpreting $H R$ values in the clinical setting. Using repeated, real-world, HR-PPG data obtained from wearables or apps data could enable physicians to provide personalized HR goals to a level that was before unattainable. ${ }^{9}$

In this study, we have shown that HR-PPG measurements are valid, and our nomograms of HR-PPG measurements obtained by patients remotely can now be interpreted by physicians, across a wide variety of patient phenotypes. These data can inform 
patients about physical fitness and could help providers offer counseling on lifestyle changes or provide overall encouragement and support based on these real-world HR norms. ${ }^{9}$

Our study has several important limitations. Our enrollment of individuals who downloaded the Instant Heart Rate app may be associated with higher socioeconomic status, technological awareness, and knowledge of elevated cardiovascular risk factors. Our validation cohort comprised of consecutive patients referred to the cardiovascular clinic differing from the general population, which could limit generalizability. However, our validation was purposefully designed to look at a broader spectrum of people who might use the app-based PPG for HR measurements, including more people with abnormal ECGs and cardiovascular disease in whom PPG might be expected to be less accurate. Despite this, we demonstrated a high validity of these measurements, in line with previously published literature.

The PPG in our data was obtained using a specific app and accuracy of measurement may vary based on different user interfaces to ensure adequate contact and signal processing algorithms that may occur in different PPG approaches. In addition, as users recorded HR-PPG measurements on demand, rather than being passively monitored, available HR-PPG do not reflect all possible real-world HR and our nomograms might not generalize to $H R$ values measured passively by wearables. In addition, we did not have the context around the measurements (i.e., food intake, post exercise, palpitations, etc.), which may have influenced the $\mathrm{HR}$ values. For example, in the "known resting HR data set", we observed an average HR $2.8 \mathrm{bpm}$ higher than in our full HR data set. One plausible explanation for this finding is that patients might be measuring their HR at rest, while having palpitations, leading to a higher upper boundary of HR in this data set and dragging the average HR higher. However, our high number of measurements collected per user in the full HR data set, combined with our large cohort size was able to describe the variability of $\mathrm{HR}$ according to age, gender, race, or BMI. Although the relationship between HR-PPG and step count confirms prior literature, our absolute values of step count may be underestimated owing to non-carrying time of the smartphone. ${ }^{36}$ Therefore, our findings should be interpreted with caution, especially in those $>8000$ average daily step counts, which represent a very small subset of participants in our study. The Health eHeart Study population is less racially, ethnically and geographically diverse and of a higher socioeconomic status than the average United States population, so care must be taken in applying these results to other populations with different characteristics. ${ }^{18,33}$ However, our population is likely representative of participants who are most likely to use this kind of technology. Owing to the cross-sectional nature of our study design, we were unable to investigate incident disease states and its relationship with HR-PPG and this should be examined in future studies. In addition, although self-reports of medical diagnoses in the $\mathrm{HeH}$ study is reliable, ${ }^{37}$ it may suffer from recall bias and social desirability biases.

Using a unique, real-world cohort that is the largest of its kind, we were able to describe the distribution of real-world HR-PPG among patients by means of remotely measured, smartphonebased PPG measurements. Our findings add granularity to the distribution of HR in specific subgroups not previously described and may assist physicians to interpret remotely obtained, realworld, on-demand, HR-PPG values measured by patients across a wide variety of patient phenotypes and medical conditions.

\section{METHODS}

\section{Study design}

We first performed an in-person validation study of the app to determine its accuracy in assessing HR-PPG. We then analyzed 3,144,332 HR-PPG signals from 66,788 participants obtained using the app as part of the Health eHeart Study in a cross-sectional population-based study.

\section{Smartphone-based PPG validation study}

In order to validate the accuracy of the app-based HR-PPG signal, we simultaneously recorded a 10-second HR-ECG and HR-PPG in 50 consecutive participants referred to Cardiology clinic at UCSF, after 5 min of rest. Mean differences were computed between successive cardiac cycles of HR-PPG and HR-ECG, in milliseconds and bpm. Rhythms were classified as being normal (sinus rhythm) or abnormal and by their regularity. Irregular rhythms included atrial fibrillation, premature ventricular, or atrial contractions and atrial flutter with variable atrioventricular block.

HR-PPG measurements were obtained using the Instant Heart Rate app (Azumio inc), a popular application for measuring HR and a smartphone's

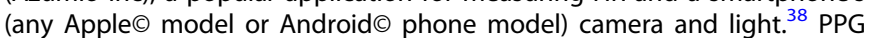
recordings are obtained "on demand" by the user steadily applying the pulp of their finger on the smartphone camera and thus is an on-demand measurement, as opposed to passive measurements made by some wearables. Participants were free to measure HR at any frequency and time of day. The study team did not provide additional instructions on when to measure such HR measurements.

\section{Population study design}

We performed a cross-sectional analysis of data obtained from 1 April 2014 and 30 April 2018 from consecutive participants enrolled in the Health eHeart $(\mathrm{HeH})$ Study - a worldwide, internet-based, longitudinal eCohort. English-speaking adults, 18 years or order, with an email address were eligible to join. ${ }^{39}$ The Health eHeart Study participants complete online surveys relating to demographics, physical activity levels, medical conditions, and medications in order to allow for the collection of patient reported outcomes and allows for connecting devices and apps (such as those that count steps) to the study. ${ }^{39}$ The study was approved by the UCSF Institutional Review Board and informed consent was obtained from all participants. For the analysis of HR-PPG, we included all Health eHeart Study participants that recorded at least one HR-PPG measurement and connected their Azumio account to the Health eHeart Study.

Participants were actively recruited through a variety of campaigns at UCSF (through clinics and electronically delivered invitations) and by partner organizations (e.g., American Heart Association), and passively recruited through word of mouth and press releases. For the first data collection set ("eVisit"), participants were asked to answer questions regarding the basic demographics, previous medical history and medications. We calculated body mass index from self-reported weight and height and classified individuals as normal weight (BMI $\geq 18.5-<25)$, overweight (BMI $25-30)$ and obese $(\mathrm{BMI} \geq 30$ ). We derived the following medication classes based on medication survey answers: beta blockers, beta agonists, amiodarone and non-dihydropyridine calcium channel blocks (CCB).

\section{Data collection}

Heart rate measures using PPG were obtained using the Instant Heart Rate (Azumio, Inc) smartphone app on either Android or iOS operating systems and the smartphone camera. Resultant changes in reflected light intensity are interpreted by an algorithm as pulsatile blood volume changes, which is then translated into HR. At least 15 seconds of PPG signal, sampled at $100-120 \mathrm{~Hz}$, were collected. Signals were processed to identify the rising edge in order to identify beat to beat intervals and calculate an average HR over the recording interval (Supplementary Fig. 1). If the underlying rhythm was an arrhythmia (atrial fibrillation, atrial flutter, premature ventricular contractions, supraventricular tachycardia), we used the peak of each HR-PPG waveform instead of the rising edge. Although it is difficult to record accurate HR measures during physical exertion, HR measurements taken immediately after a physical activity are possible.

\section{Weighting of repeated HR measurements}

To account for repeated measures, HR and 24-hour step counts were logtransformed to approximate normality and the geometrical mean for each participant was calculated. ${ }^{40}$ To obtain the weights, we used linear mixed models with random intercepts to estimate the ICC of the repeated logtransformed measures. Then the weight for each participant was 
calculated as $\left(\right.$ weight $\left._{i}=\frac{N_{i}}{1+I C C\left(N_{i}-1\right)}\right)$, where $N i$ is the number of repeated HR measures for participants $I$. The denominator of the weight represents the inflation of the variance of the participant-specific means owing to the correlation of the repeated measures. In a final step, the weights were normalized to sum to the number of participants. If the repeated measures were independent $(I C C=0)$, then participants would be weighted in proportion to their number of observations; at the other extreme, if the repeated measures were perfectly correlated $(I C C=1)$, then the geometric mean for each participant would be given equal weight. We then used weighted linear models to examine the independent correlates of geometric mean HR.

\section{Statistical analysis}

Continuous variables are presented using mean \pm standard deviation (SD) or median (interquartile range) and were compared using the $t$ test, the Mann-Whitney test or one-way analysis of variance, as appropriate. Categorical variables are presented as frequencies (percentages) and compared using either Chi-square or Fisher's exact tests. For our validation study, we estimated ICC for agreement between HR-ECG values obtained in clinic, using a 12-lead ECG, with the HR-PPG obtained using the Azumio app, at the heart rate level and at the signal level, by comparing averaged $\mathrm{R}-\mathrm{R}$ intervals between both methods. We also used a Bland-Altman plot to assess agreement between the simultaneous HR-PPG and HR-ECG recordings. ${ }^{41}$

To clean the data, we excluded outliers defined as values of HR-PPG outside of the biologically plausible range of $20-220 \mathrm{bpm} .{ }^{16}$ Next, to better limit the data to values most likely to be true resting values (not affected by physical activity), we created a "known resting HR data set". To do this, we restricted our HR-PPG measurements to participants who had accumulated between 10 and 25 steps during the $30 \mathrm{~min}$ prior to their HR-PPG measurements, assuming that $>25$ steps represented the lower limit of any exercise in the last $30 \mathrm{~min}$, whereas $<10$ steps might reflect users who set the phone down while exercising. We excluded participants with a medical condition from this data set. For our analyses, we summarized the repeated HR-PPG measurements for each participant, within our "known resting HR data set" and the "full HR data set" using weighted geometric means. As a measure of dispersion of the geometric mean HR-PPG, we used $95 \%$ prediction intervals, accounting for both the standard error of the overall mean and the residual variation of the participant-specific geometric means. We used a non-parametric kernel regression method to create centile charts for heart rate and step counts with respect to age, gender, and step count.

In the subgroup with at least one medical condition, we used unadjusted and age-adjusted HR-PPG to examine the independent associations of comorbidities with geometric mean HR-PPG. Univariable linear regression models were fitted to describe the relationship between age, gender, race/ethnicity, body mass index (BMI), height, weight, step count, and number of medical conditions. Furthermore, two multivariable linear regression models were fitted to examine the associations between HR and demographics or comorbidities, after assessing for heteroscedasticity and multicollinearity between the variables. The first included age, gender, race/ethnicity, number of medical conditions, BMI and step count, and the second included age, gender, individual medical conditions, medication use (beta blockers, amiodarone, beta agonists, and CCB). In an exploratory analysis presented in the appendix, selected interactions between medical conditions and medications were also included. Furthermore, we calculated person-based HRV, in beats per minute, by deriving the standard deviation of R-R intervals of HR-PPG within users with more than two recordings. We then fitted two multivariable regression models to examine the relationship between HRV and age, gender, medical conditions, BMI, and step count. We also derived average values of real-world HR-PPG and HRV based on time of day, based on weekend vs weekdays and based on seasons.

Two-tailed $p$ values $<0.01$ were considered statistically significant, without further correction for multiple testing. Statistical analyses were performed using STATA 15.1 (College Station, TX) and python 2.7 with packages scientific python version 0.19.1, scikit learn version 0.19.0.

\section{Reporting summary}

Further information on research design is available in the Nature Research Reporting Summary linked to this article.

\section{DATA AVAILABILITY}

The data that support the findings of this study are available on request from the corresponding author (J.O.). The data are not publicly available due to them containing information that could compromise participant privacy/consent.

\section{CODE AVAILABILITY}

Our code is available at https://github.com/robertavram-md/Real-world-HR-norms.

\section{ACKNOWLEDGEMENTS}

David J. Wen for extracting the Health eHeart data.

\section{AUTHOR CONTRIBUTIONS}

R.A., J.O., and E.V. analyzed and interpreted the data. M.B., R.R., N.W. recruited the patients for the in-clinic validation. R.A. and J.O. drafted the manuscript. All authors revised the content of the manuscript, contributed significantly to its improvement, read and approved its final version. J.O. takes responsibility for all aspects of the reliability and freedom from bias of the data presented and their discussed interpretation.

\section{ADDITIONAL INFORMATION}

Supplementary information accompanies the paper on the npj Digital Medicine website (https://doi.org/10.1038/s41746-019-0134-9).

Competing interests: G.M. has received research funding from Medtronic and Cardiogram Inc, is a consultant for Lifewatch and InCarda, and holds equity in InCarda. P.K. is an employee of Azumio. There are no disclosures for the remaining authors. Azumio provided no financial support for this study and only provided access to the heart rate data. Data analysis and interpretation was performed independently from Azumio.

Publisher's note: Springer Nature remains neutral with regard to jurisdictional claims in published maps and institutional affiliations.

\section{REFERENCES}

1. Kannel, W. B., Kannel, C., Paffenbarger, R. S. J. \& Cupples, L. A. Heart rate and cardiovascular mortality: the Framingham Study. Am. Heart J. 113, 1489-1494 (1987).

2. Gillum, R. F. Epidemiology of resting pulse rate of persons ages 25-74-data from NHANES 1971-74. Public Health Rep. 107, 193-201.

3. Mason, J. W. et al. Electrocardiographic reference ranges derived from 79,743 ambulatory subjects. J. Electrocardiol. 40, 228-234 (2007).

4. Pierdomenico, S. D., Bucci, A., Lapenna, D., Cuccurullo, F. \& Mezzetti, A. Clinic and ambulatory heart rate in sustained and white-coat hypertension. Blood Press. Monit. 6, 239-244 (2001).

5. Mancia, G. et al. Effects of blood-pressure measurement by the doctor on patient's blood pressure and heart rate. Lancet 2, 695-698 (1983).

6. Korshoj, M. et al. The relation of ambulatory heart rate with all-cause mortality among middle-aged men: a prospective cohort study. PLOS ONE 10, e0121729 (2015).

7. Chan, P. H. et al. Diagnostic performance of a smartphone-based photoplethysmographic application for atrial fibrillation screening in a primary care setting. J. Am. Heart Assoc. 5, e003428 (2016).

8. Elgendi, M. On the analysis of fingertip photoplethysmogram signals. Curr. Cardiol. Rev. 8, 14-25 (2012).

9. Khan, J. S., Sharma, A. \& Seth, P. Resting heart rate and wearable technology. CMAJ 188, 755-755 (2016).

10. Johansen, C. D. et al. Resting, night-time, and $24 \mathrm{~h}$ heart rate as markers of cardiovascular risk in middle-aged and elderly men and women with no apparent heart disease. Eur. Heart J. 34, 1732-1739 (2013).

11. Lequeux, B., Uzan, C. \& Rehman, M. B. Does resting heart rate measured by the physician reflect the patient's true resting heart rate? White-coat heart rate. Indian Heart J. 70, 93-98 (2018).

12. Hansen, T. W. et al. Prognostic superiority of daytime ambulatory over conventional blood pressure in four populations: a meta-analysis of 7,030 individuals. $J$. Hypertens. 25, 1554-1564 (2007).

13. Piccione, G., Giannetto, C., Assenza, A., Casella, S. \& Caola, G. Influence of time of day on body temperature, heart rate, arterial pressure, and other biological 
variables in horses during incremental exercise. Chronobiol. Int. 26, 47-60 (2009).

14. Liao, D. et al. Age, race, and sex differences in autonomic cardiac function measured by spectral analysis of heart rate variability-the ARIC study. Atherosclerosis Risk in Communities. AJC 76, 906-912 (1995).

15. Tanaka, H., Monahan, K. D. \& Seals, D. R. Age-predicted maximal heart rate revisited. JACC 37, 153-156 (2001).

16. Fox, S. M. 3, Naughton, J. P. \& Haskell, W. L. Physical activity and the prevention of coronary heart disease. Ann. Clin. Res. 3, 404-432 (1971).

17. Arnold, J. M., Fitchett, D. H., Howlett, J. G., Lonn, E. M. \& Tardif, J.-C. Resting heart rate: a modifiable prognostic indicator of cardiovascular risk and outcomes? Can. J. Cardiol. 24, 3A-8A (2008).

18. Molfino, A. et al. Body mass index is related to autonomic nervous system activity as measured by heart rate variability. Eur. J. Clin. Nutr. 63, 1263-1265 (2009).

19. Palatini, P. \& Julius, S. Elevated heart rate: a major risk factor for cardiovascular disease. Clin. Exp. Hypertens. 26, 637-644 (2004).

20. Dominguez-Rodriguez, A., Blanco-Palacios, G. \& Abreu-Gonzalez, P. Increased heart rate and atherosclerosis: Potential implications of ivabradine therapy. WJC 3, 101-104 (2011).

21. Palatini, P. et al. Reproducibility of heart rate measured in the clinic and with 24hour intermittent recorders. Am. J. Hypertens. 13, 92-98 (2000).

22. Molfino, N. A. et al. Assessment of airway tone in asthma. Comparison between double lung transplant patients and healthy subjects. Am. Rev. Respir. Dis. 148, 1238-1243 (1993).

23. Simonsson, B. G., Jacobs, F. M. \& Nadel, J. A. Role of autonomic nervous system and the cough reflex in the increased responsiveness of airways in patients with obstructive airway disease. J. Clin. Investig. 46, 1812-1818 (1967).

24. Shah, P. K. D., Lakhotia, M., Mehta, S., Jain, S. K. \& Gupta, G. L. Clinical dysautonomia in patients with bronchial asthma. Chest 98, 1408-1413 (1990).

25. van Gestel, A. J. \& Steier, J. Autonomic dysfunction in patients with chronic obstructive pulmonary disease (COPD). J. Thorac. Dis. 2, 215-222 (2010).

26. Kerkhof, P. L. M., Kuznetsova, T., Yasha Kresh, J. \& Handly, N. Cardiophysiology illustrated by comparing ventricular volumes in healthy adult males and females. Adv. Exp. Med Biol. 1065, 123-138 (2018).

27. Ryan, S. M., Goldberger, A. L., Pincus, S. M., Mietus, J. \& Lipsitz, L. A. Gender- and age-related differences in heart rate dynamics: are women more complex than men? JACC 24, 1700-1707 (1994).

28. Gaar, K. A. J. Cardiac output: is there a sex difference? Med. Hypotheses 23, 149-152 (1987).

29. Kirkup, A. S. A. L. A direct comparison of wet, dry and insulating bioelectric recording electrodes. Physiol. Meas. 21, 271 (2000).

30. O'Toole, M. L. Gender differences in the cardiovascular response to exercise. Cardiovasc. Clin. 19, 17-33 (1989).
31. Bathula, R., Francis, D. P., Hughes, A. \& Chaturvedi, N. Ethnic differences in heart rate: can these be explained by conventional cardiovascular risk factors? Clin. Auton. Res. 18, 90-95 (2008).

32. Martins, D., Tareen, N., Pan, D. \& Norris, K. The relationship between body mass index, blood pressure and pulse rate among normotensive and hypertensive participants in the third National Health and Nutrition Examination Survey (NHANES). Cell Mol. Biol. 49, 1305-1309 (2003).

33. Resting Heart Rate \& Your Health. blog.fitbit.com (2016). Available at: https://blog. fitbit.com/what-your-resting-heart-rate-can-reveal-about-your-health/.

34. Smulyan, $\mathrm{H}$. et al. Influence of body height on pulsatile arterial hemodynamic data. JACC 31, 1103-1109 (1998).

35. Chapman, J. H. Profound sinus bradycardia in the athletic heart syndrome. J. Sports Med. Phys. Fitness 22, 45-48 (1982).

36. Amagasa, S. et al. How well iPhones measure steps in free-living conditions: cross-sectional validation study. JMIR Mhealth Uhealth 7, e10418 (2019).

37. Wang, J. B. et al. Cigarette and e-cigarette dual use and risk of cardiopulmonary symptoms in the Health eHeart Study. PLOS ONE 13, e0198681 (2018).

38. Mitchell, K., Graff, M., Hedt, C. \& Simmons, J. Reliability and validity of a smartphone pulse rate application for the assessment of resting and elevated pulse rate. Physiother. Theory Pract. 32, 494-499 (2016).

39. Olgin, J. E., Pletcher, M. J. \& Marcus, G. M. Thbe Health-eHeart study. Health eHeart Study Available at: https://www.health-eheartstudy.org/. (Accessed: 18 May 2017).

40. Manikandan, S. Measures of central tendency: median and mode. J. Pharmacol. Pharmacother. 2, 214-215 (2011).

41. Bland, J. M. \& Altman, D. G. Statistical methods for assessing agreement between two methods of clinical measurement. Lancet 1, 307-310 (1986).

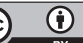

Open Access This article is licensed under a Creative Commons Attribution 4.0 International License, which permits use, sharing, adaptation, distribution and reproduction in any medium or format, as long as you give appropriate credit to the original author(s) and the source, provide a link to the Creative Commons license, and indicate if changes were made. The images or other third party material in this article are included in the article's Creative Commons license, unless indicated otherwise in a credit line to the material. If material is not included in the article's Creative Commons license and your intended use is not permitted by statutory regulation or exceeds the permitted use, you will need to obtain permission directly from the copyright holder. To view a copy of this license, visit http://creativecommons. org/licenses/by/4.0/.

(c) The Author(s) 2019 